Technological University Dublin

DƯBLIN

ARROW@TU Dublin

\title{
Theoretical Analysis of a Volume Holographic Lens Using Matlab
}

\author{
Sanjay Keshri \\ Technological University Dublin, sanjaykumar.keshri@tudublin.ie \\ Kevin Murphy \\ Technological University Dublin, kevin.p.murphy@tudublin.ie \\ Izabela Naydenova \\ Technological University Dublin, izabela.naydenova@tudublin.ie
}

See next page for additional authors

Follow this and additional works at: https://arrow.tudublin.ie/cieocon2

Part of the Electromagnetics and Photonics Commons, and the Optics Commons

\section{Recommended Citation}

S. K. Keshri, K. Murphy, I. Naydenova and S. Martin, "Theoretical Analysis of a Volume Holographic Lens Using Matlab," 2019 Photonlcs \& Electromagnetics Research Symposium - Spring (PIERS-Spring), 2019, pp. 2814-2818, doi: 10.1109/PIERS-Spring46901.2019.9017426.

This Conference Paper is brought to you for free and open access by the Centre for Industrial and Engineering Optics at ARROW@TU Dublin. It has been accepted for inclusion in Conference Papers by an authorized administrator of ARROW@TU Dublin. For more information, please contact arrow.admin@tudublin.ie, aisling.coyne@tudublin.ie,gerard.connolly@tudublin.ie. Funder: Technological University Dublin; FOCAS Research Institute

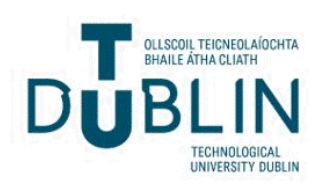




\section{Authors}

Sanjay Keshri, Kevin Murphy, Izabela Naydenova, and Suzanne Martin

This conference paper is available at ARROW@TU Dublin: https://arrow.tudublin.ie/cieocon2/40 


\title{
Theoretical Analysis of a Volume Holographic Lens Using Matlab
}

\author{
Sanjay Kumar Keshri, Kevin Murphy, Izabela Naydenova, and Suzanne Martin \\ Centre for Industrial and Engineering Optics/School of Physics, College of Sciences and Health \\ Technological University Dublin, Kevin Street, Dublin 8, Ireland
}

\begin{abstract}
Volume holographic lenses have great potential for different types of applications requiring light redirection and beam shaping such as solar light collection and LED light management. For lighting applications using LEDs, it is essential to make a highly efficient optical element to be placed in front of the LED in order to decrease energy losses. For that reason, a careful theoretical analysis of the properties and operation regime of the lens must be carried out at the design stage. The characteristics of focusing Holographic Optical Elements (HOE) depend on many factors including their thickness, spatial frequency, the angular range of incidence of the incoming light, recording wavelength and replay wavelength. Simulation of lens performance that takes into account the above parameters is critical for designing experimentally efficient HOEs, including for LED applications. In this paper, the parameters of a volume cylindrical holographic lens have been analyzed by using Kogelnik Coupled Wave Theory and Bragg's Law. Simulations have been carried out in Matlab to define the regime of operation of a model unslanted grating and cylindrical holographic lens and discussed in the context of possible limitations imposed by recording the optical element in a photosensitve material. This theoretical analysis is very useful in designing lenses to avoid production of multiple beams and lowered efficiency. The analysis can also help in devising strategies to overcome these limitations and in achieving diffraction efficiency approaching $100 \%$.
\end{abstract}

\section{INTRODUCTION}

Holographic Optical Elements (HOEs) could play a valuable role in lighting applications including solar light concentrator [1] and solid-state lighting [2]. HOEs are lightweight and compact elements which could be used to tailor the light to specified shapes and directions [3]. For each lighting application, there are certain challenges in the fabrication of HOEs, especially for LED lighting applications. Since the performance of such elements depend on many parameters including HOE thickness, spatial frequency, slant angle, the angle of incidence and wavelength of the illuminating light and up to date they have been mostly used with narrow band polarised sources such as lasers.

Recently, research has demonstrated a number of useful applications of holographic optical elements in light management. For example, a surface diffractive lens has been used to control the luminous intensity distribution of LED light [4], a volume hologram has been utilized as a solar concentrator $(f=14 \mathrm{~cm})$ to concentrate sunlight onto a photovoltaic cell [5] and a volume hologram has been demonstrated as a color demultiplexer to split a white LED into its component colours at three different positions $(f=50 \mathrm{~cm})[6]$. Recently work, has demonstrated efficient collimation of light from a green LED by utilizing a low spatial frequency optical element, but such elements don't allow for large angles of light redirection [3]. A variation in diffraction efficiency was observed experimentally across a cylindrical holographic lens aperture in the literature $[2,3]$. Uniformity was improved by altering the exposure conditions to allow time for diffusion across the larger fringe periods. During this work it was also experimentally observed that, since the spatial frequency of the holographic element varies across the lens aperture, care had to be taken not to let the spatial frequency drop below a threshold that would produce multiple diffracted orders. Thus, it is a valuable exercise to carry out a theoretical simulation of the expected lens performance before carrying out further experimental work. This will optimize the design so that for the desired photopolymer layer thickness, the HOE will remain in the 'thick' operation regime across the whole lens aperture, ensuring that the device outputs a single diffracted beam.

So far there is no published numerical simulation work which uses the well-known $Q$ and $\rho$ (rho) parameters introduced into the literature by Klein \& Cook [7] and Moharam et al. [8]. to models the regime of operation for locations characterised by different spatial frequencies for a cylindrical holographic lens fabricated for broader wavelength light source. These parameters have been shown to be useful guidelines in determining the operational regimes (i.e., thick, thin or mixed regime) of holographic devices. In this paper, numerical simulations based on the $Q$ and $\rho$ have been carried out in a specially written Matlab program [9] using Kogelnik Coupled Wave Theory (KCWT) [10] and Bragg's Law [11] to calculate the characteristics of the studied HOE. 


\section{Q AND $\rho$ SIMULATION FOR GRATING}

A HOE operation regime can be significantly different depending on whether it is considered as a thick, thin or mixed operation regime of a hologram and this has traditionally been determined by the $Q$ value [7] which is defined by Eq. (1)

$$
Q=\frac{2 \pi \lambda d}{n \Lambda^{2}}
$$

where, $\lambda$ = wavelength of the illuminating beam, $d=$ thickness of recording medium, $n=$ average refractive index of the medium, $\Lambda=$ grating spacing. For a large value of $Q(>10)$, the grating operation regime is considered as a thick and for the value of $Q(<1)$, the grating operation regime is considered as thin [7]. The operation regime between $1<Q<10$ is a mixed regime. The $Q$ parameter is a normalized measure of thickness which is used for defining whether a hologram is in the thick, thin or mixed operation regime, and when there is a limitation on grating strength. $Q$ is more reliable in devices which are recorded with low index modulation. Table 1 shows the characteristics of thick, thin and mixed grating operation regimes.

Table 1. Characteristics of the thick, intermediate (mixed) and thin operation regimes of a grating.

\begin{tabular}{|c|c|c|}
\hline Thin regime & Mixed regime & Thick regime \\
\hline$Q<1$ or $\rho<1$ & $1<Q<10$ or $1<\rho<10$ & $Q>10$ or $\rho>10$ \\
\hline $\begin{array}{c}\text { Described by Raman-Nath theory } \\
\text { Multiple orders of diffraction }\end{array}$ & Intermediate region & $\begin{array}{c}\text { Described by Kogelnik's Coupled } \\
\text { Wave Theory (KCWT) One } \\
\text { first order of diffraction }\end{array}$ \\
\hline Broader angular selectivity & Intermediate angular selectivity & Narrow angular selectivity \\
\hline $\begin{array}{c}\text { Efficiency in first-order }<33.4 \% \\
\text { Multiple beams }\end{array}$ & Multiple order beams may appear & $\begin{array}{l}\approx 100 \% \text { efficiency possible } \\
\text { in a single diffracted order }\end{array}$ \\
\hline
\end{tabular}

The $\rho$ (rho) parameter is also used to define the grating operation regime and is considered more reliable in devices which are recorded with higher index modulation. $\rho$ is defined as [8]

$$
\rho=\frac{\lambda^{2}}{\Lambda^{2} n \Delta n}
$$

where, $\lambda=$ vacuum wavelength of the light, $\Lambda=$ grating spacing, $n=$ mean refractive index of the photosensitive medium and $\Delta n=$ amplitude of the sinusoidal modulation of the refractive index. For a large value of $(\rho>10)$, the grating operation regime is considered as a thick and for the value of $(\rho<1)$, the grating operation regime is considered as thin [8]. The regime operation between $1<\rho<10$ is a mixed regime. Table 1 shows the regime of the grating according to $Q$ and $\rho$ values.

Figure 1(a) presents a simulation of the $Q$ parameter values calculated for a wide range of the grating thicknesses and spatial frequencies. The simulation is carried out using Eq. (1) at constant refractive index $(n=1.5)$ and wavelength of illumination $(532 \mathrm{~nm})$. In Fig. 1(a), the grating is in thick operation regime $(Q>10)$ everywhere, except for the orange colour region. For example, at a thickness of $50 \mu \mathrm{m}$ in Fig. 1(a), the grating must be recorded at a spatial frequency $>300 \mathrm{lines} / \mathrm{mm}$ to be in the thick operation regime. Fig. 1(b) presents a simulation of $\rho$ for a range of refractive index modulations and grating spatial frequencies. This simulation is carried out using Eq. (2) at a constant average refractive index of the medium (for photopolymer, $n=1.5$ ) and a wavelength of illumination $(532 \mathrm{~nm})$. In this simulation, the black colour on the left of colour map represents the thick operation regime of grating according to Moharam et al. [8]. Fig. 1(b) shows, for a grating with a spatial frequency of 300 lines $/ \mathrm{mm}$, it is necessary to record it with index modulation $<2 * 10^{3}$ in order to ensure that it is in the thick operation regime. In the thin and mixed operation regime, there will be more diffracted orders and these simulations will be helpful to avoid thin or mixed regime to get an efficient grating or lens for applications like LED.

Using a thickness-angular plane a simulation has also been performed, shown in Fig. 2(a), for an unslanted grating at a spatial frequency of 300 lines $/ \mathrm{mm}$. For each thickness, the refractive index modulation value has been chosen to give $100 \%$ efficiency at that thickness. The simulation provides the maximum efficiency at each thickness at different Bragg angle and angular selectivity of the curve decreases as thickness increases. Threshold thickness for $Q=10, \rho=10, Q=1, \rho=1$ 


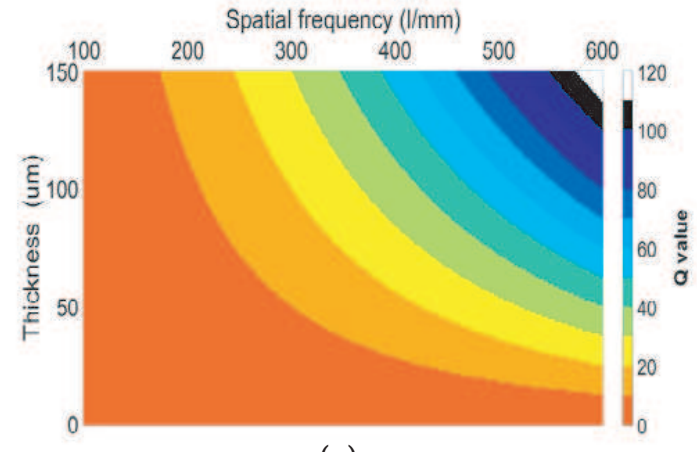

(a)

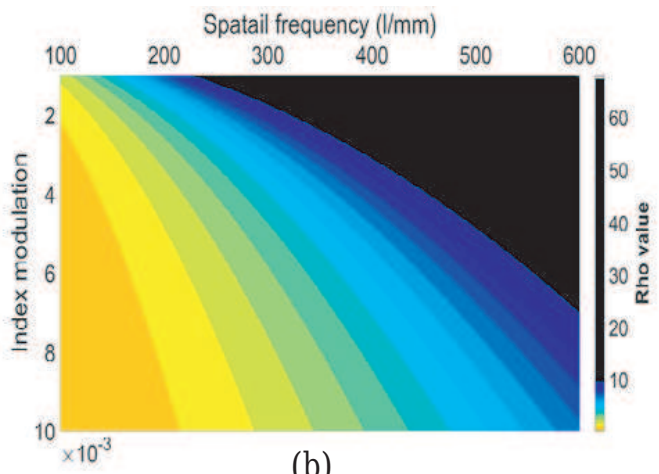

(b)

Figure 1. (a) Thickness-spatial frequency plane with a colour map of $Q$ value (b) Index modulation-spatial frequency plane with a colour map of $\rho$ value.

have also been highlighted so that the thin, mixed and thick operation regime of the grating can be identified according to the $Q$ and $\rho$ parameters.

In Fig. 2(b) a spatial frequency-angular plane has also been provided at a thickness of $75 \mu$ assuming $100 \%$ efficiency at each spatial frequency at different Bragg angle. It can be seen in Fig. 2(b) that as the spatial frequency increases the Bragg angle increases. In this simulation, the threshold spatial frequencies for $Q=10, \rho=10, Q=1, \rho=1$ have also been marked to identify where the thin, mixed and thick operation regimes occur. Fig. 2 has been modeled using Kogelnik Coupled Wave Theory which is valid only for thick operation regime $(Q>10$ or $\rho>10)$ and only serves to identify where multiple beams may occur. Once the thin or mixed regime is reached the values shown are no longer meaningful. In both Fig. 2(a) and Fig. 2(b) the $Q$ parameter is the more appropriate parameter as the index modulation is low. For $Q<10$, it is not in thick regime and the regime for $Q<1$ can only be accurately modeled using the Raman-Nath equation. In the next section, this type of simulation has been done to define the thick and thin operation regime of a cylindrical holographic lens.

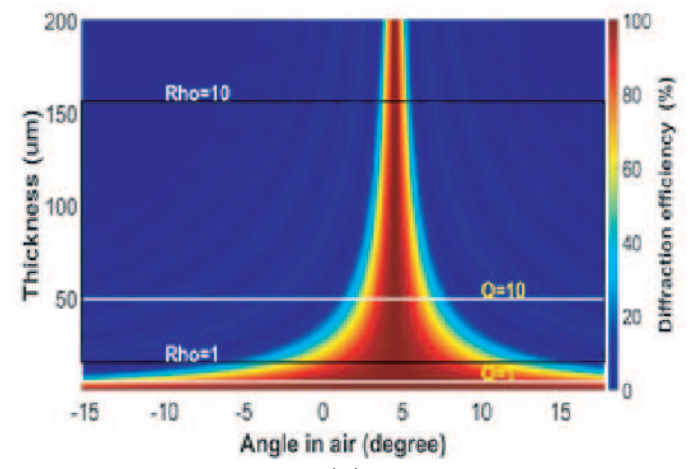

(a)

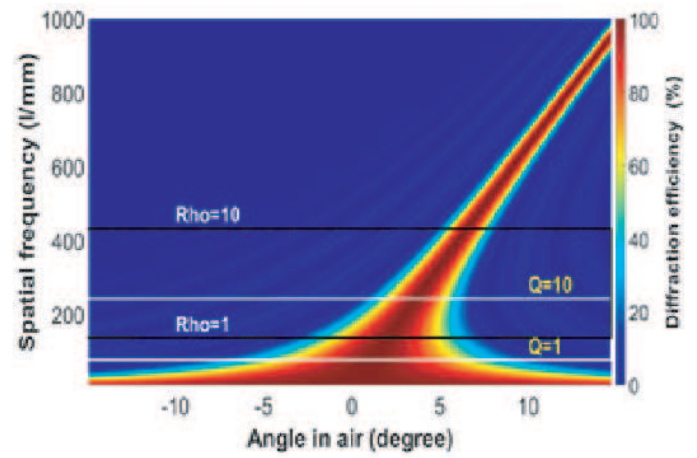

(b)

Figure 2. (a) Thickness-spatial frequency plane with a colour map of $Q$ value at a fixed spatial frequency of $300 \mathrm{l} / \mathrm{mm}$ (b) Index modulation-spatial frequency plane with a colour map of $\rho$ value at a fixed thickness of $75 \mu \mathrm{m}$.

\section{SIMULATION FOR CYLINDRICAL HOLOGRAPHIC LENS}

A simulation for a cylindrical holographic lens with the same parameters as that fabricated in literature [3] has been carried out. Fig. 3 is a simulation plane for a cylindrical holographic lens of the thickness of $75 \mu \mathrm{m}$. It shows the variation of efficiency at each lateral position and angular positon. The Bragg angle changes for each lateral position of the lens. The recording scheme for this holographic lens and the lateral positions are outlined in Fig. 4. In Fig. 3 the extreme edges of the lens (red lines) fabricated by Keshri et al. [3] are outlined with the thresholds of $Q$ and $\rho$ defining the thick, mixed and thin operation regimes. According to the $Q$ parameter, the lateral lens positions from $+7 \mathrm{~mm}$ to -1.5 only can be considered as purely in the thick operation regime 
and the grating is actually in the thin operation regime for about $1 \mathrm{~mm}$ close to the right edge. For the $\rho$ parameter only the lateral lens positions from $+7 \mathrm{~mm}$ to $+3 \mathrm{~mm}$ are truly operating in the thick regime, however, this device was recorded with a low index of modulation so it is more appropriate to decide on the operation regime using the $Q$ parameter. Experimentally, some very weak higher order beams were observed even though an overall diffraction efficiency of $66.5 \%$ was nevertheless achieved in the device. Therefore it can be seen that this type of simulation could be useful in designing a highly efficient grating or HOE and it is clear that further modelling and experimental investigation of the intermediate regime would be useful.

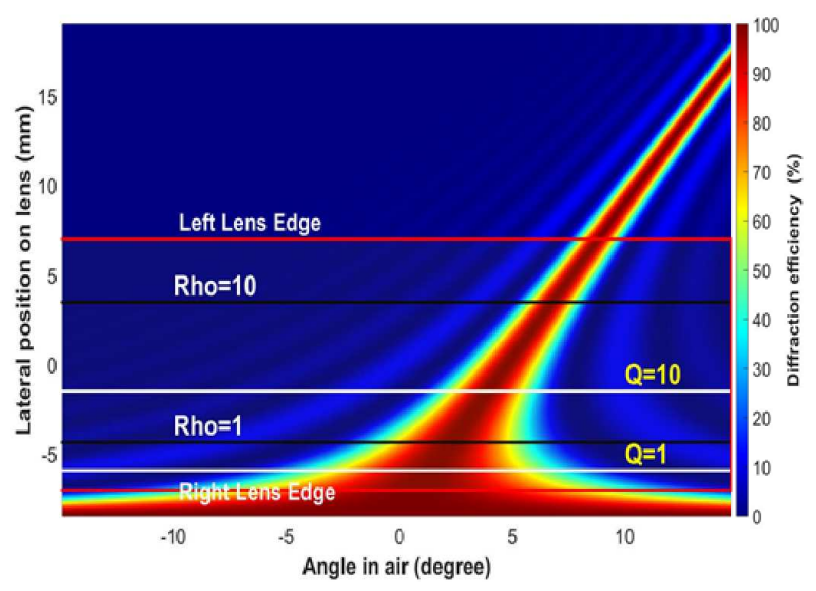

Figure 3. The lateral position-angular plane with a colour map of efficiency for a holographic cylindrical lens recorded at a central frequency of 300 lines $/ \mathrm{mm}$ and at a fixed thickness of $75 \mu \mathrm{m}$.

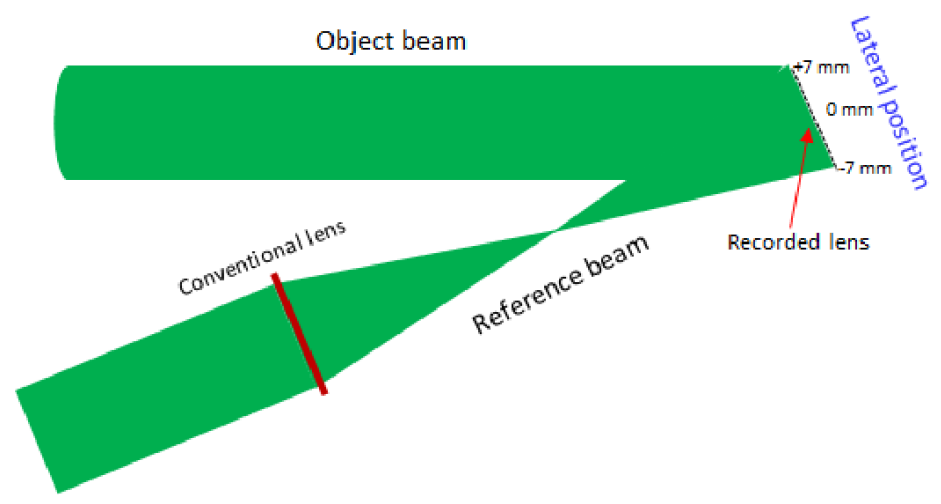

Figure 4. Schematic of recording a holographic lens of diameter $14 \mathrm{~mm}$ (with the lateral lens positions marked).

\section{CONCLUSION}

HOEs can play a valuable role as devices in many broadband applications, including for solar concentrators and LED lighting. A HOE operating in the thin operation regime produces multiple orders can be modelled using the Raman-Nath equation, while a thick operation regime HOE which produces only one first order can be modelled by KCWT. Because the diffraction efficiency can reach $100 \%$ a "thick" HOE can conceivably be used in front of LEDs in lighting applications to make an overall extremely efficient luminaire device. However, the fabrication of HOEs operating in the thick operation regime depends not only on its thickness but also on recording parameters such as the beam wavelength, spatial frequency, and index modulation. The $Q$ and $\rho$ parameters can be used to identify the thick and thin operation regimes of operation of the HOE. A simulation developed using Matlab shows the different parameters of gratings and a cylindrical holographic lens specifying the thick and thin operation regimes according to the $Q$ and $\rho$ parameters. This simulation also highlights a potential contributor to losses [3]. From the above simulations it can 
be seen that the regime the fabricated lens operates varies across the lens aperture. The grating structure will function in the thick and intermediate operation regime across most of the lens aperture, but it does cross into the thin regime along one edge. This is highly dependent on the particular diffractive lens design and so should be modeled for each new lens in order to test the limitations and choose the best recording arrangement. These simulations could be very useful in the design and fabrication of highly efficient gratings or HOEs for any application requiring either a broad angular or spectral range.

\section{FUNDING}

Technological University Dublin (Fiosraigh Dean of Graduate Students Award); Enterprise Ireland (CF20144622, IP20170590); FOCAS Research Institute.

\section{ACKNOWLEDGMENT}

The authors wish to thank, FOCAS Research Institute, Technological University Dublin Fiosraigh Postgraduate Scholarship Fund, and Enterprise Ireland Technology Development Fund.

\section{REFERENCES}

1. Marín-Sáez, J., J. Atencia, D. Chemisana, and M.-V. Collados, "Characterization of volume holographic optical elements recorded in Bayfol HX photopolymer for solar photovoltaic applications," Opt. Express, Vol. 24, A720-730, 2016.

2. Keshri, S., K. Murphy, V. Toal, I. Naydenova, and S. Martin, "Comparison of two methods for equalising the diffraction efficiency of different spatial frequency components of holographic optical elements," Proc. SPIE, Holography: Advances and Modern Trends V, 102330X, 2017.

3. Keshri, S., K. Murphy, V. Toal, I. Naydenova, and S. Martin, "Development of a photopolymer holographic lens for collimation of light from a green light-emitting diode," Appl. Opt., Vol. 57, No. 22, E163, doi:10.1364/AO.57.00E163, 2018.

4. Motogaito, A. and K. Hiramatsu, "Fabrication of binary diffractive lenses and the application to LED lighting for controlling luminosity distribution," Opt. Photonics J., Vol. 3, 67-73, March 2013.

5. Collados, M. V., D. Chemisana, and J. Atencia, "Holographic solar energy systems: The role of optical elements," Renew. Sustain. Energy Rev., Vol. 59, 130-140, 2016.

6. Shen, Z., T. Lan, L. Wang, and G. Ni, "Color demultiplexer using angularly multiplexed volume holograms as a receiver optical end for VLC based on RGB white LED," Opt. Commun., Vol. 333, 139-145, 2014.

7. Hariharan, P., "Basics of holography," Thin Thick Holograms, 33-34, Cambridge Cambridge Univ. Press, doi10.1017/CBO9780511755569.004, 2002.

8. Moharam, M. G. and L. Young, "Criterion for bragg and Raman-Nath diffraction regimes," Vol. 17, No. 11, 1757-1759, 1978.

9. Register, A. H., "A guide to MATLABß) object-oriented programming," SciTech Publishing Inc., Boca Raton, 2007.

10. Kogelnik, H., "Coupled wave theory for thick hologram gratings," The Bell System Technical Journal, Vol. 48, No. 9, 2909-2947, 1969.

11. Jauncey, G. E. M., "The scattering of x-rays and Bragg's law," Proc. Natl. Acad. Sci., Vol. 10, 57-60, USA, 1924. 